\title{
Boundary Detection Robots
}

\author{
Hussein Safa, Ahmad Taha Abdulsadda, Ahmad Gahnam Wadday
}

\begin{abstract}
Mobile robot sensors have appeared as techniques for tracking the environment, search and rescue, exploration and mapping, civil infrastructure analysis, and military operations. Discovery and tracking of polluted areas with sensor mobile multi-robots is now regarded the solution to environmental and human safety issues. This paper shows some algorithms intended to allow mobile multi robots with sensor to estimate and monitor for polluted an irregular area in a synchronized way. Changes in the behavior of dangerous environmental boundaries, such as fire spreads or oil spills, provide appropriate data to mitigate the issue or even support evacuation actions to save human or animal life. In this paper, we present a model using a two-robot called it Boundary Detection Robot (BDR) moving around an environmental boundary to predict its shape through a continuous analytical function based on the combination of polynomial approximation. These robots are composed of many sensors each with embedded processors, wireless communication, and movement capabilities. We explain that when we increase the sample frequency and the robot velocity, the strategy converges to the exact boundary. We conducted experiments with simulated and actual robots to assess the estimation quality. We analyze the reliability of the control unit and other component in a robot simulator and assess the efficiency of the all components in a realistic set-up and environment model. We implement a stable transmission range of robot control laws with sensors to track irregular area boundaries.
\end{abstract} robotics, control theory.

\section{INTRODUCTION} $\mathrm{T}_{\text {he big towns and increasing industrial regions pose a high }}$ risk of different kinds of pollutants affecting the quality of life of society. The discovery and monitoring of contaminated fields using autonomous mobile robots is now often seen as a solution to environmental and human safety problems. Wireless sensor networks Installed on a robot have provoked the interest of specialists from different areas by imposing a large number of theoretical and practical challenges related to their implementation. Like these robots, the effectiveness of current conventional pollution prevention

Revised Manuscript Received on February 05, 2020.

* Correspondence Author

Hussien Safa*, Communication department, Al najaf Technical engineering College, Al Furat Al Awast Technical University (ATU), Al najaf City, Iraq.

Ahmad Taha Abdulsadda, Communication department, Al najaf Technical engineering College, Al Furat Al Awast Technical University(ATU), Al najaf City, Iraq. abdulsad@msu.edu. Technical Engineering College, Al Furat Al Awast Technical University(ATU), Al najaf City, Iraq.

(c) The Authors. Published by Blue Eyes Intelligence Engineering and Sciences Publication (BEIESP). This is an open access article under the CC BY-NC-ND license (http://creativecommons.org/licenses/by-nc-nd/4.0/)
Keywords :Boundary detection, Environmental monitoring,

Ahmad Gahanam Wadday, Communication Department, Al Najaf

systems can be enhanced. The objective is to convert the robot with wireless sensor networks into an adaptive sensor system with smart conduct instead of fixed wireless sensor networks see fig1.1.A. This robots can be used to detect and monitor polluted areas or spill regions that obtained and represented in Specific shape due to controlled environmental parameters see fig.(1).

On the other hand, during the last decade there is a lot of research about the description this areas or regions. In the 19th century, it was known how the polygon enclosed in a convex body was geometrically characterized, minimizing the area difference between itself and the convex body enclosed. On the other side, the geometric characterization of a polygon, which involves a defined strictly convex body, is more complicated and less intuitive. In the 19th century it was also shown that the approximation error for various useful metrics goes to zero as $1 / \mathrm{P}^{2}$ for a convex planar body, where $\mathrm{P}$ is the number of vertices of the interpolating polygon. As detailed in research [1] -[4].

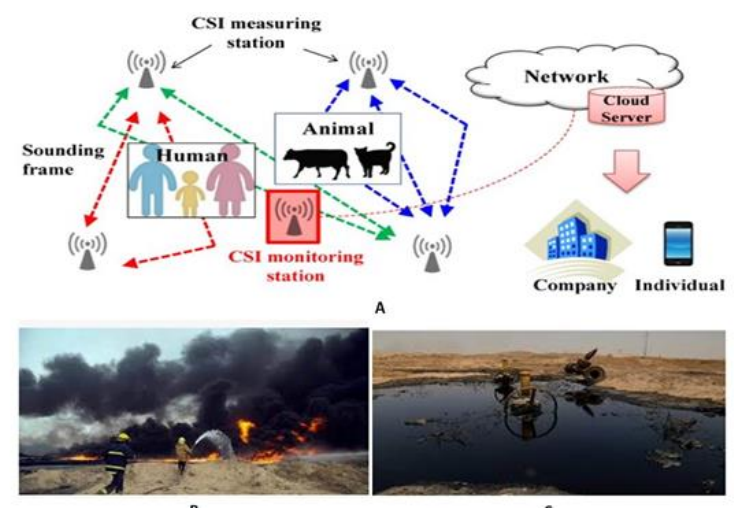

Figure 1: Describe fixed wireless sensor networks, describe polluted areas or spill regions.

IN 2006, the researchers for robotic sensor networks is to explore the feasibility of using multiple low-altitude short endurance (LASE) or unmanned air vehicles (UAVs) to monitor and track the boundaries of large forest fires in a cooperative way. Monitoring a fire's perimeter by using a multi-UAV decentralized strategy. It is presumed that the UAVs have limited range of communication and sensing. The researchers was unable to accurately determine the irregular area and the unmanned air vehicles (UAVs) return to the base station for further shipment Leads to it takes a long time to fully cover the irregular area in typical time . which is supported by researcher [4]-[8].

IN 2007, the researchers describes a cooperative mobile robot network that can detect and track a perimeter defined in the environment by a certain chemical spill. 


\section{Boundary Detection Robots}

The experiments of boundary Tracking for small area and it was verified with static parameters while dynamic perimeter tracking was verified in Matlab. Which is proposed by researcher [9]. IN 2007, the problem of estimating and tracking boundaries by automated sensors is emerging. The common goal is to develop a distributed algorithm that allows a limited number of mobile robot with sensor networks to detect and estimate the boundaries of a region as it evolves. That's what the researcher worked on [10]-[13].

in this paper, We suppose a new robotic platform call boundary detection robot (BDR), was developed with ubiquitous modularity in software, hardware, and control systems as a goal. The driving part of the base is intended to allow the use of distinct drive technologies without changing the primary controller to detect and monitor polluted environmental limits. Additionally it is tied to specific sensors but the sensor shield allows different configurations and sensing modalities to be switched out based upon the desired application. Our algorithm does not involve any previous data about the polluted environment boundary dynamics and confirms our submission through tests with actual multi-robots from (BDR). We experimentally demonstrate with multi-robots that our technique can estimate and predict non-convex limits for any region or environment, even with incorrect measurements.

The rest of the paper is organized as: In section II gives a detailed explanation with the equations for the mathematical formulation of non-convex and irregular area as software's, and Explain the hardware component for robot in detailSection III presents the algorithms for the robot to detect and monitor the non-convex area in different shapes and build up the steps for the algorithms that are used in Matlab program to implement. Finally, section IV presents the simulation results and discussion ,verification of analytical results for the robot when detect and monitor the non-convex and irregular area in different shapes.

\section{HARDWARE COMPONENT AND CONVEX BODIES THEOREM PROCEDURE FOR PAPER SUBMISSION}

This section seeks to discuss the different Sensors and Control System Hardware characteristics in higher depth. In terms of function and abilities, each element is defined. Concepts are described that are essential for a practical comprehension of the hardware. The tests that were performed on the Hardware aspects of the Sensors and Control System are described in chapter 3, along with the results of the tests, and the conclusions drawn described in chapter 4 . We will discuss two main parts making up each robot namely the sensors and the robot structure.

\section{A. Sensors}

The boundary representation for collecting the relevant information from the environment is an significant component of the assignment. To do this, depending on the specific implementation of use, a wide variety of sensors can be used.

There will be two sorts of data the robot must collect, namely the situation and position. The situation data is associated with the robot's own status, such as temperature, amount of wheel rotations, or battery level. The position information is collected from the environment such as the distance between the robot (BDR) and the amount of points to represent the environment boundary.

Author (s) can send paper in the given email address of the journal. There are two email address. It is compulsory to send paper in both email address.

\section{B. Wheel Encoders}

The IR sensors and gyroscope sensors allow the robot to measure direction and angle, respectively. With them, a certain amount of degrees can be turned to select a robot target. To calculate the distance along the boundary, the rotary encoders are used to obtain this data from the start of the first point on this boundaries and the end of the last point. In this paper, they are referred to as wheel encoders when attached to the motors controlling the robot's wheels. Rotary encoders are mechanical elector instruments creating performance proportional to a shaft or axle's angular position: they can be incremental or absolute. The absolute rotary encoders can detect a disk's precise position.

The incremental model, on the other hand, can only determine the direction of the shaft movement, clockwise or counterclockwise. The required information in this paper refers to the amount of times the wheel of the robot turned. Once this information is supplied, the extent to which the robot moved forward or backward can be calculated. In this paper, the incremental rotary encoder was used to get this data.

\section{The robot structure}

The design of the robot (BDR)consists of three primary robot components: the chassis containing the wheels and their motors, the Arduino board and the batteries. This primary platform is linked to all the remaining hardware components used in the robot. The company Adriano details the metal chassis many types that the robot uses. The robot car is equipped with two rough wheels and one motor to regulate each. This model is especially interesting because each wheel is attached to a suspension which offers wider angles of motion so that they can move through uneven surfaces. Fig. (2) shows the robot vehicle components

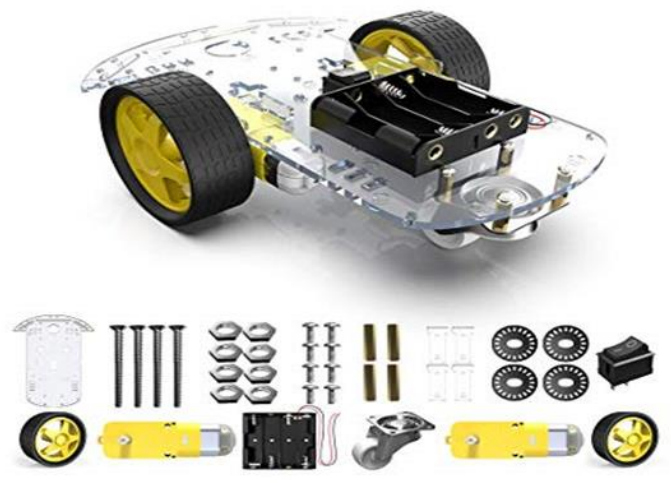

\section{THE ROBOTS USING THE PSEUDO-DISTANCE}

In this section we modify an algorithm leading a group of $\left(\mathrm{R}_{\mathrm{i}}\right)$ robots to continually calculate and update a slowly moving boundary estimate. The estimate for any area is calculated as an interpolating polygon.

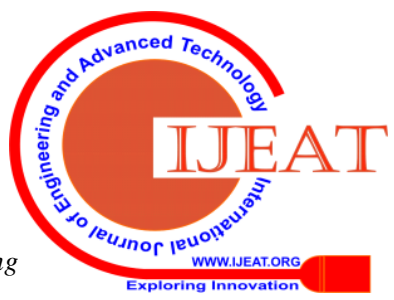


The purpose of the algorithm is to position the interpolation ii. points in such a way that they are distributed uniformly at points according to the pseudo-distance (DP). As discussed in the Introduction, we assume that

- Initially, the robots have a boundary estimate.

- Each robot has a sensor and algorithms to evaluate locally the boundary's tangent and curvature.

- The robots share information with the base station via a iv. communication network of ring-topology.

We let $\left\{R_{i}\right\}$ when $i \in\{1, \ldots, m R\}$ be the positions of the mobile robots and we let $\left\{P_{j}\right\}$ when $j \in\{1, \ldots, n P\}$ be the vertices points of the interpolating polygon; in a practical implementation, We suppose that a copy of these virtual positions is maintained by each robot. Based on the initial estimation the boundary of the area, We add extra assumptions as follows at the time $\mathrm{t}=0$, the robots reached an allocation point $\partial \mathrm{B}$ and the interpolation points are distributed (possibly uniformly) at the estimated boundary. We suppose that both the interpolation points and the robots are ordered and distributed counterclockwise, and along this boundary that the robots (BDR) movement counterclockwise with speed $\mathrm{S}_{\mathrm{n}}$, see fig.(3).

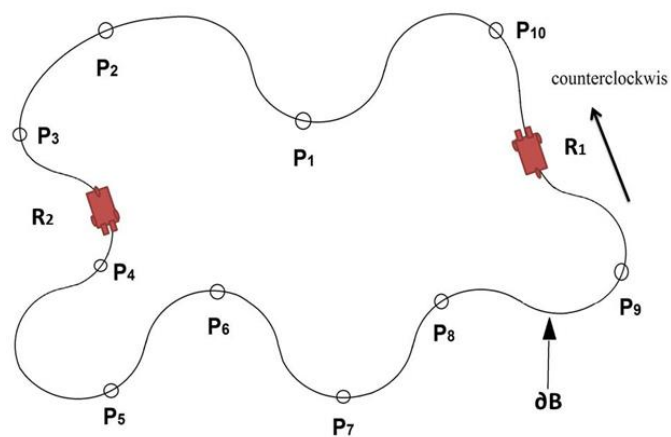

Figure 3: Boundary of irregular area.

In the fig. (3),the boundary is represent by the solid line $\partial \mathrm{B}$, the triangles are the robots, the circles are the points of interpolation, and the dotted line is the approximate polygon defined by the points of interpolation. The robots have two goals:

(i) update the interpolation points in such a way that they are distributed uniformly along $\partial \mathrm{B}$ in accordance with the estimated pseudo-distance of $D_{p}$,

(ii)the robots move along the boundary equally distributed according to arc length distance.

To achieve these two objectives we propose to use Estimate Update and Pursuit Algorithm that can be summarized as steps:

- Every robot (BDR) moves counterclockwise along the time-varying

- collects estimates of the boundary and of its tangent and curvature

- Using these estimates, the Robot (BDR) completes the following four actions:

i. Each Robot (BDR) updates the positions of the interpolation points so that they take value in the boundary $\partial \mathrm{B}$. In other words, as sufficient information is available, each interpolation point $\left\{P_{j}\right\}$ when $j \in\{1, \ldots, n P\}$, is projected onto the measured boundary.

After an interpolation point $\left\{\mathrm{P}_{\mathrm{j}}\right\}$ has been projected, the Robot (BDR) collects sufficient information so that it can locally optimize its position along the estimate of $\partial \mathrm{B}$.

iii. Every Robot (BDR) estimates the arc length distance between itself and its immediate clockwise and counterclockwise neighbors and uses this information to speed up or slow down.

At last, the updated interpolation point $\left\{\mathrm{P}_{\mathrm{j}}\right\}$ is transmitted to appropriate neighboring Robot.

The first two steps combine the affect of finding and updating the boundary's local estimates. The third step has the affect of uniformly distributing robots (BDR) across the boundary. The fourth step has the effect of making the robot (BDR) keeping correctly distributed boundary estimate information.

\section{SIMULATION AND EXPERIMENTAL RESULTS}

We send one of robots to explore the desired regular Circle. In this experiment we calculate the speed, the time the robot needs and how many points it used to represent the irregular area. During the passage of the robot (R1) from the middle of the area (X1)to its surroundings (Q). Always these points are processing from robot. After processing that is done on this point the robot sends the coordinates to the base station. The result of this experiment is received from the base station in the MATLAB program as shown in fig. (4). The methodology used to calibrate circuit movements was to use the IR distance sensor on the robot board to achieve accurate real-world measurements of speed and number of points. In practice, the robot can be directed to the area from any position within it, so that the robot's speed and number of points can be calculated by distinguishing between distance measurements over time. The number of points received as a result is shown in the following figure on the computer screen of MATLAB as shown in fig. (5).

We can be compared result in the fact for irregular area with the results shown on the MATLAB program for several parameter, with Note the Length of area $(300 \mathrm{~cm})$ and width of area $(200 \mathrm{~cm})$. These results to show more clearly can be summarized in the following table (1).

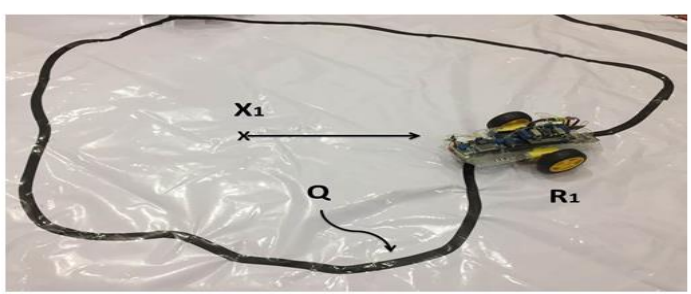

Figure 4: Robot moves on irregular area.

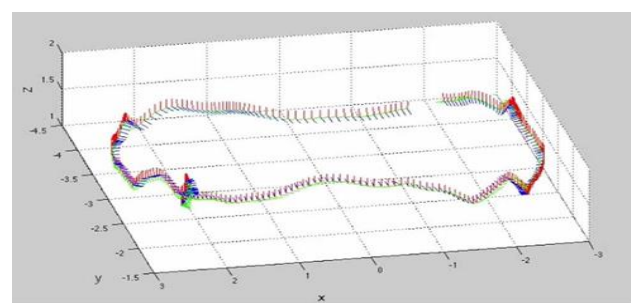

Figure 5: Robot moves on irregular area from MATLAB program. 
Boundary Detection Robots

Table 1: Actual and simulated value of the speed and time.

\begin{tabular}{|c|c|c|c|}
\hline Status & speed & Time & No. of Points \\
\hline Actual & $0.19 \mathrm{rad} / \mathrm{sec}$ & $33 \mathrm{sec}$ & $300 \times 200 \mathrm{~cm}$ \\
\hline Simulation & $0.165 \mathrm{rad} / \mathrm{sec}$ & $38 \mathrm{sec}$ & 50 point \\
\hline
\end{tabular}

\section{DISCUSSION AND CONCLUSION}

Distributed coverage for irregular area is a difficult issue for multi-robot systems in various situations. In order to execute sensing, tracking, information collection, search, or distributed service tasks, the objective is to deploy a group of robots spreading and moving around a polluted region. Line regular Coverage, line irregular Coverage, circle Boundary Coverage and irregular area. Boundary coverage by one or two robot is variation of the issue of coverage studied in this paper. we present the real issue of two-robot coverage and discover of an irregular area's boundaries and the space within it to distinguish it from outside. The two-robot (BDR) have apportionment in a irregular boundary area as arrange for discover it and the communication between two-robot each other and base station through wireless unit also. Finally, the boundary as Matlab program map representation was established and developed to model the working space and the algorithm for irregular area detection of boundaries. The base station receives the point as an information from the two-robot (BDR) about detecting an event early for the boundary and representation the irregular area.

\section{REFERENCES}

1. P. M. Gruber. "Approximation of convex bodies". In P. M. Gruber and J. M.Willis, editors, Convexity and its Applications, pages 131-162. Birkh“auser Verlag, 1983.

2. P. M. Gruber. "Aspect of approximation of convex bodies". In P. M. Gruber and J. M. Willis, editors, Handbook of Convex Geometry, volume A, pages 319-345. Elsevier, Oxford, UK, 1993.

3. D. W. CASBEER, D. B. KINGSTON,R. W. BEARD* and T. W. MCLAIN, "Cooperative forest fire surveillance using a team of small unmanned air vehicles" International Journal of Systems Science, Vol. 37, No. 6, 15 May 2006, 351-360

4. J. Clark, and R. Fierro, "Mobile robotic sensors for perimeter detection and tracking", Transactions,Elsevier ,May 2006

5. D. Salda na, A. Montoya, and D.Ovalle, "A Multi-Agent Model to Control Robotic Sensor Networks", IEEE, 31 December 2012

6. D. Salda na, A. Montoya, and D.Ovalle, "Improved Algorithm for Perimeter Tracking in Robotic Sensor Networks",IEEE 04 February 2013

7. S. Li, Y. Guo and B. Bingham, "Multi-robot Cooperative Control for Monitoring and Tracking Dynamic Plumes”, 2014 IEEE International Conference on Robotics \& Automation (ICRA) Hong Kong Convention and Exhibition Center May 31 - June 7, 2014. Hong Kong, China

8. J. Brink, "Boundary tracking and estimation of pollutant plumes with a mobile sensor in a low-density static sensor network", Elsevier, Urban ClimateVolume 14, Part 3, December 2015, Pages 383-395

9. M. Fahad, N. Saul, Y. Guo, and B. Bingham, "Robotic Simulation of Dynamic Plume Tracking by Unmanned Surface Vessels", 2015 IEEE International Conference on Robotics and Automation (ICRA) Washington State Convention Center Seattle, Washington, May 26-30, 2015

10. D. Salda na, R. Assunc ao, and M. F. M. Campos, "Predicting Environmental Boundary Behaviors with a Mobile Robot" IEEE Robotics and Automation Letters, (Volume: 1 , Issue: 2 , July 2016 )P: 1133 - 1139,27 January 2016

11. D. Salda na1, R. Javanmard Alitappeh2, L. C. A. Pimenta2, R Assunc ao, and M. F. M. Campos, "Dynamic Perimeter Surveillance with a Team of Robots", 2016 IEEE International Conference on Robotics and Automation (ICRA)Stockholm, Sweden, May 16-21, 2016

12. D. Salda na, R. Assunc ao, M. Ani Hsieh1, Mario F. M. Campos, and V. Kumar, "Cooperative Prediction of Time-Varying Boundaries with a
Team of Robots", IEEE,2017 International Symposium on Multi-Robot and Multi-Agent Systems (MRS), Conference: 11 January 2018

13. S. A. Ahmed, V. L. Popov, A. V. Topalov, and N. G. Shakev, "Environmental monitoring using a robotized wireless sensor network", Springer, May 2018, Volume 33, Issue 2, pp 207-214

\section{AUTHORS PROFILE}

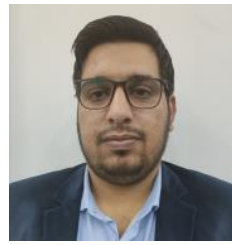

Hussein Safa Dakhil, received the B. Sc. degree in communication technical engineering from Al Furat Al Awast Technical University, Iraq in 2012 Currently he is a candidate to pursue a M. Sc. degree in communication technical engineering from Al Furat Al Awast Technical university since 2017.

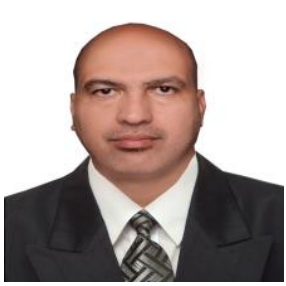

Dr.Ahmad T. Abdulsadda, received the B. Sc degree in electrical engineering from Tickrit University, Iraq in 1997, M. Sc. degree in electrical engineering from Baghdad University, Iraq in 2000, and the PHd in Electrical and Computer Engineering Department at Michigan State University, Michigan, USA. . From 2000 to 2006, he was a faculty member at Baghdad University, Iraq. Since 2006, he has been a faculty member at Technical Najaf College, Al Furat $\mathrm{Al}$ Awast University, Iraq. Currently, he is an assistance dean of Al Najaf Technical engineering college, Al Furat Al Awast Technical University. His research interests include robotics fish, feedback control systems, nonlinear estimation techniques, and control theory.

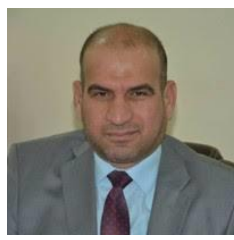

Dr.Ahmed Ghanim Wadday, received B.Sc. degree in Communication Engineering from University of Technology Al-Rasheed College , Iraq in 1991, M.Sc. degree in Communications Engineering from University of Technology Al-Rasheed College , Iraq in 1997. Ph.D. degree in Electromagnetic Fields and Microwaves Technology from University of Basrah , Iraq in 2012. From 2004 to 2014, he has been a faculty member University of Baghdad in Iraq. From 2014 to 2019, he was a Vice president of Al Furat Al Awast technical university. 